\title{
Jennifer Gloria Lowpez Story
}

Told by Jennifer Gloria Lowpez Written by Ruth DyckFehderau

Translated by Louise Blacksmith

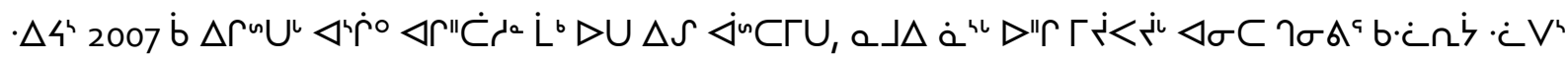

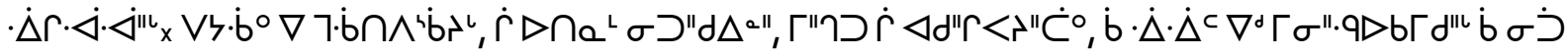

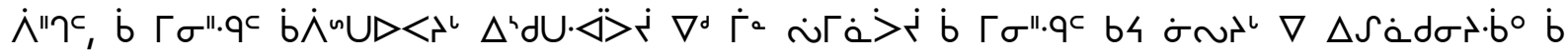

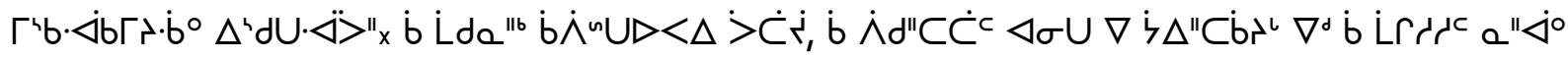

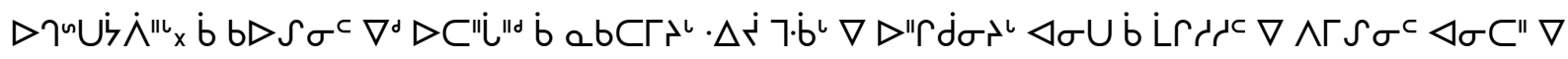
$\cdot \dot{\Delta} \sigma^{\prime \prime C} \dot{b} r^{\imath} \triangleleft \sigma \dot{r} \Gamma \sigma^{\prime \prime} \cdot 9 \triangleright b \Gamma d \dot{d} x$

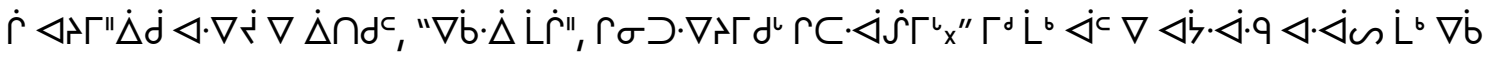

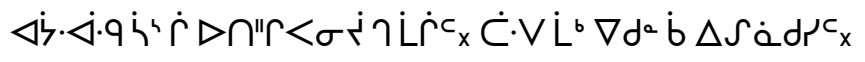

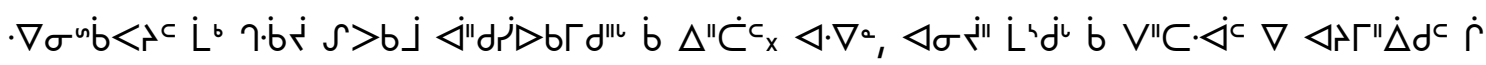
टं"i்

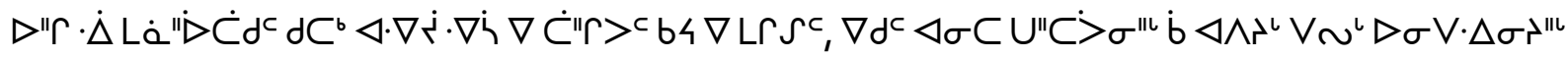

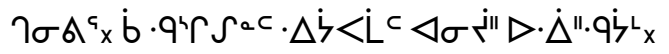

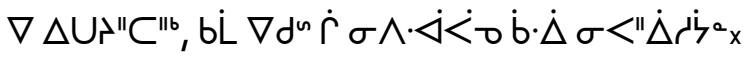

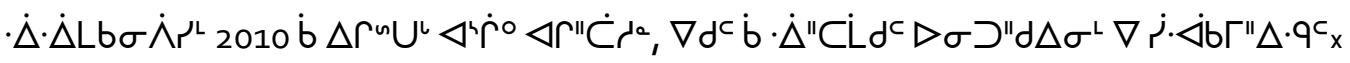

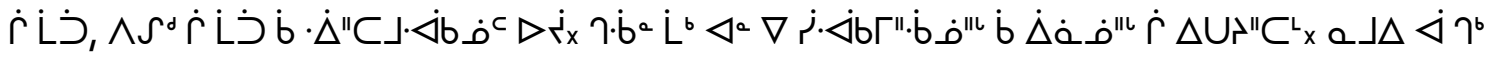

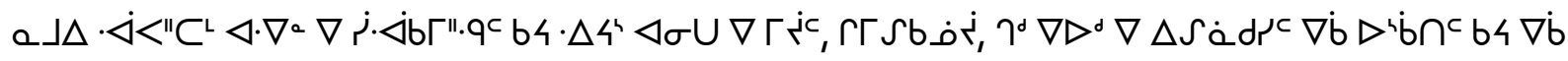

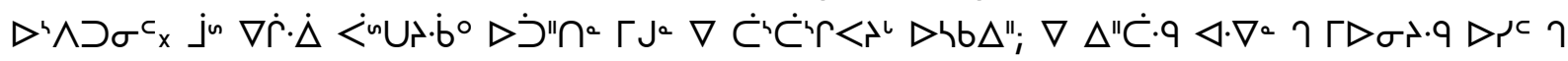

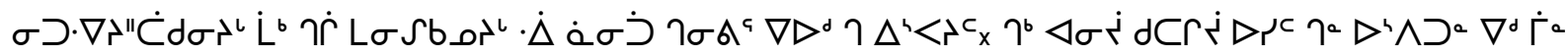

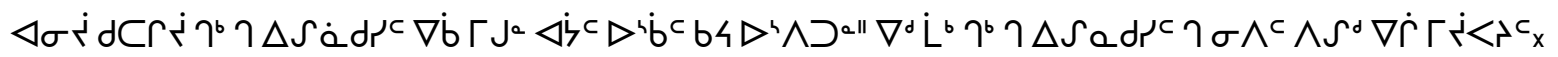

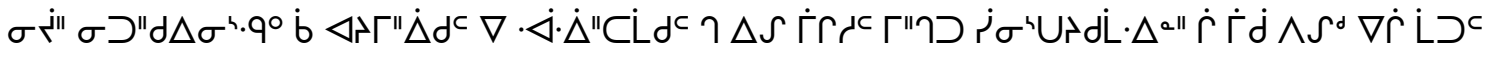

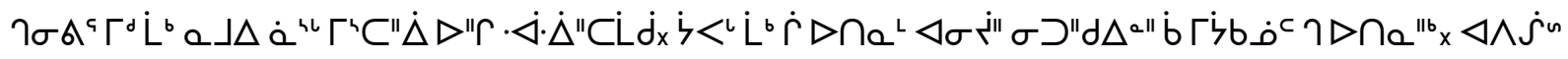

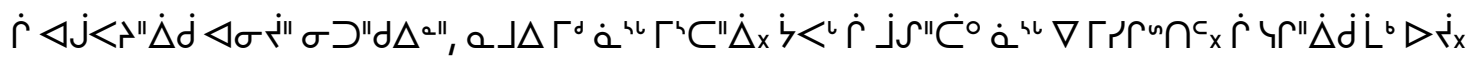

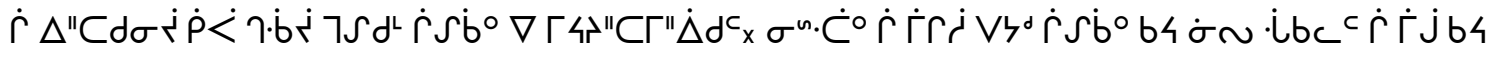

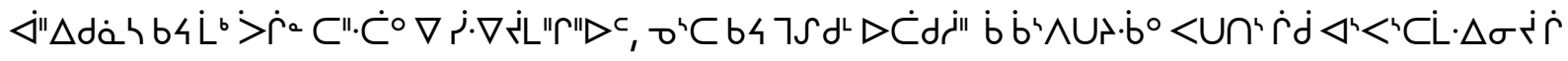




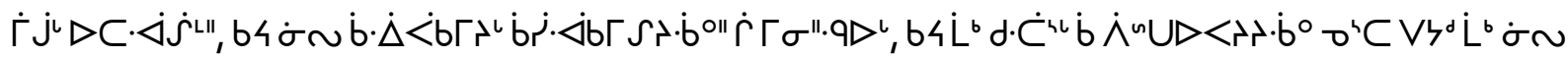

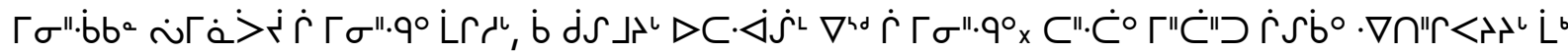

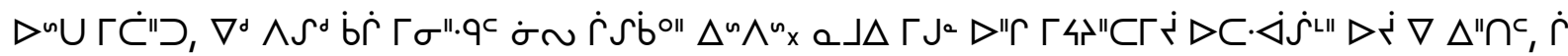

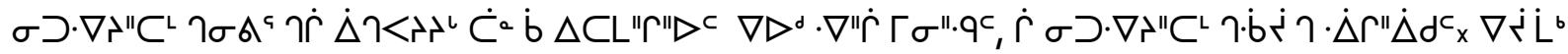

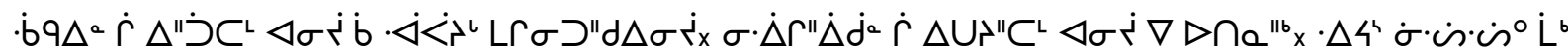

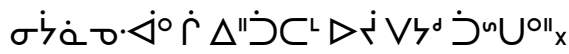

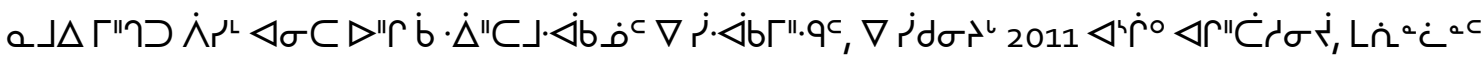

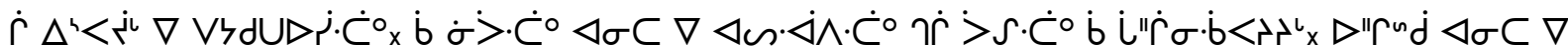

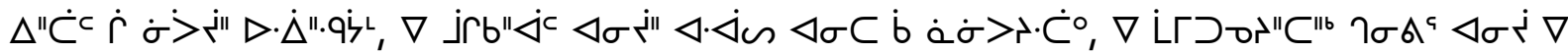

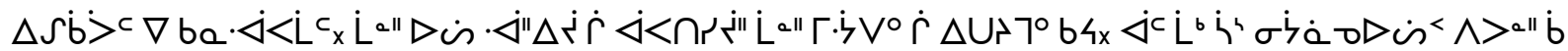

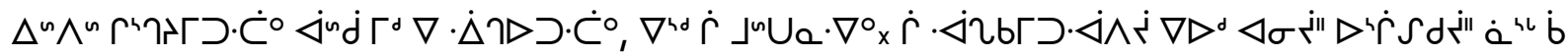
$\dot{\Gamma} \triangleright \circ\lrcorner \cdot \dot{j} c_{x}$

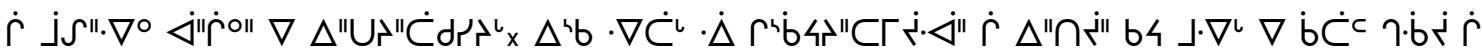

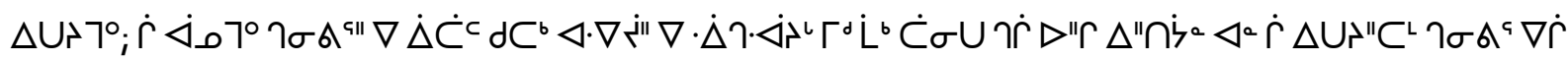

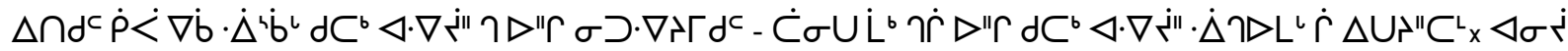

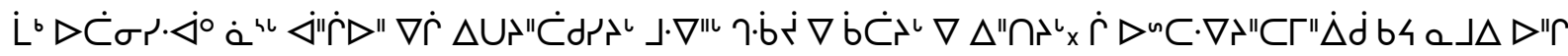

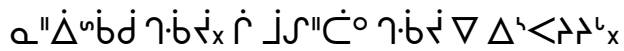

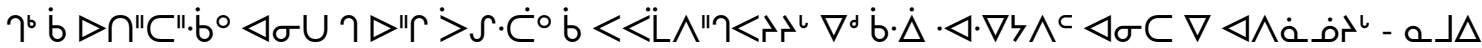

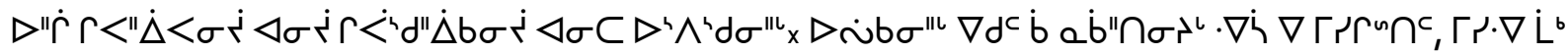

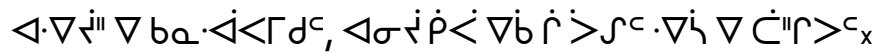

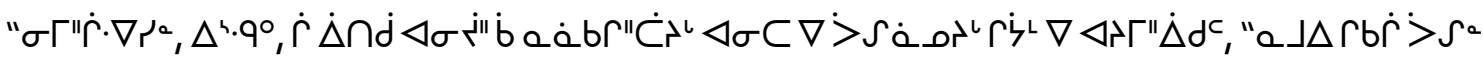

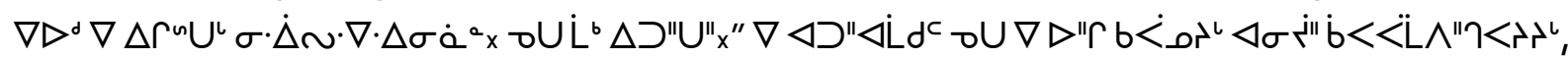

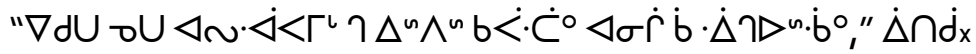

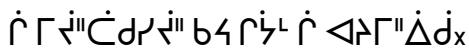

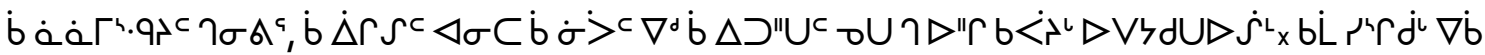

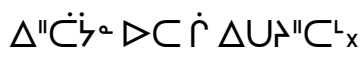

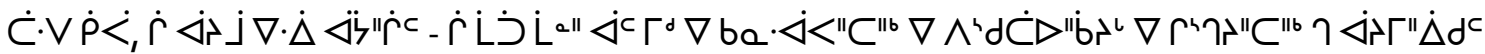

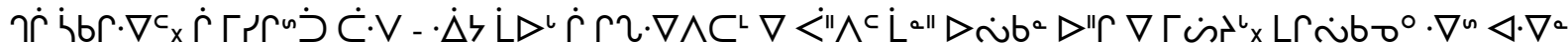

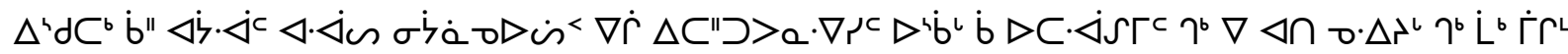

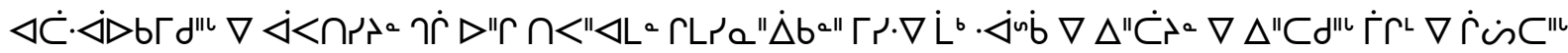

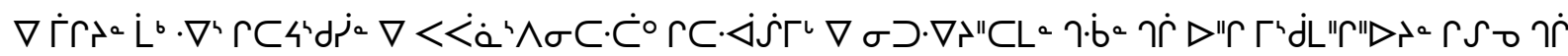




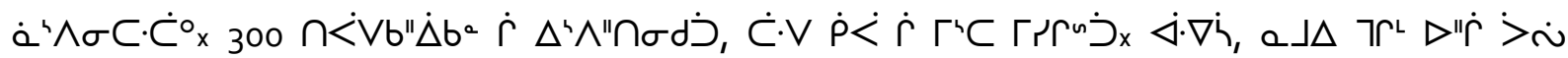

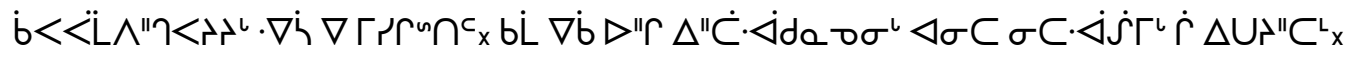

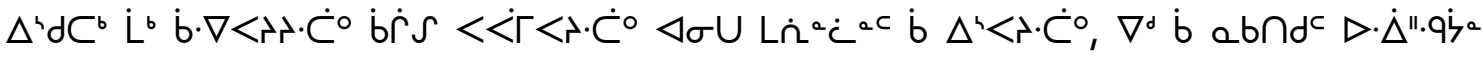

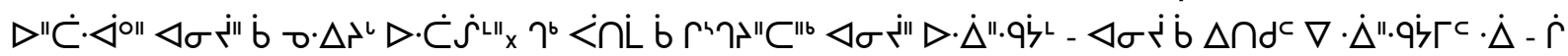

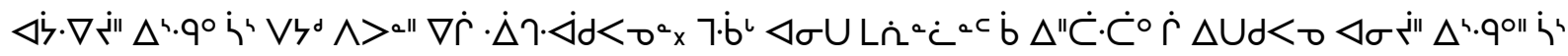

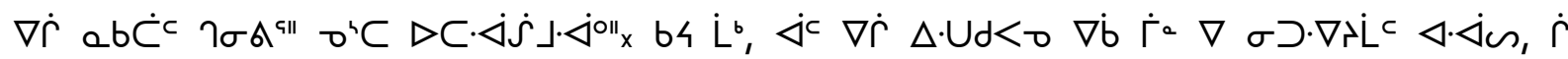

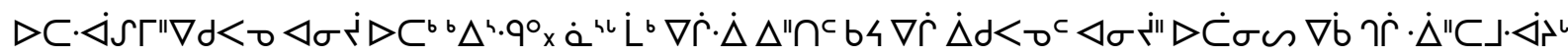
$\triangleright \dot{b} \cdot \dot{\Delta} d C^{b} \Delta^{4} \cdot q^{\circ} \nabla \cdot \dot{\Delta} \cdot \cdot \dot{<c} x$

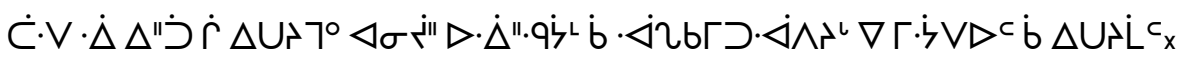

$\dot{\alpha} " \dot{C} \cdot \vee \dot{r} \triangleleft \dot{\gamma} \Gamma " \dot{\Delta} \dot{d} \triangleright \dot{r} x$

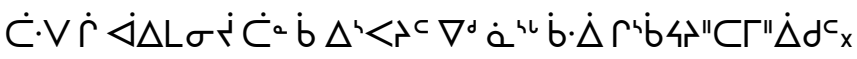

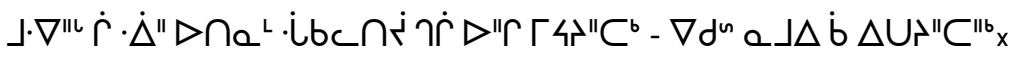

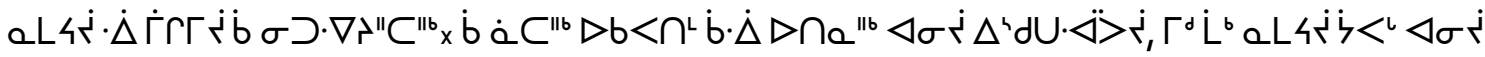

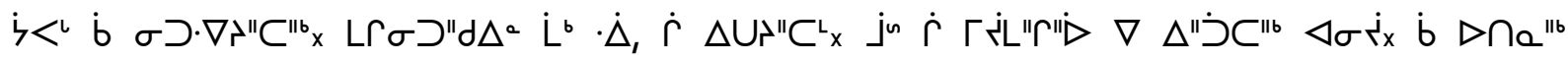

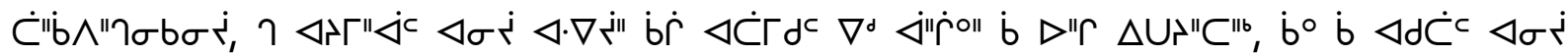

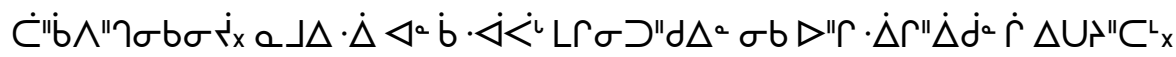

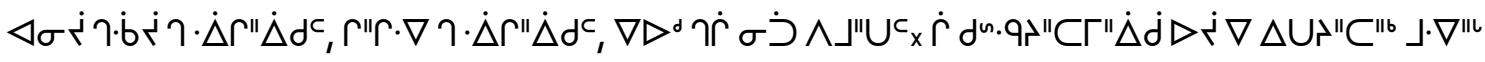

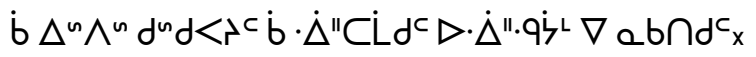

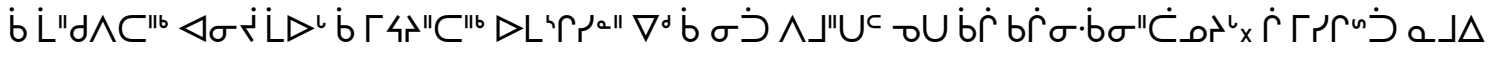

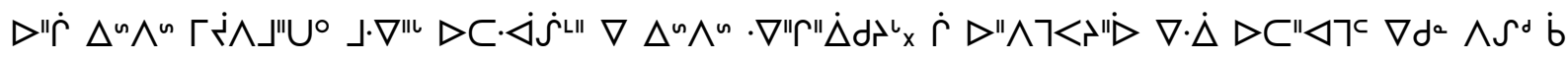

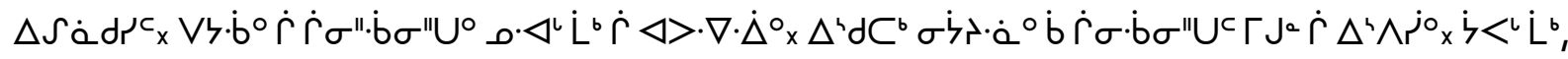

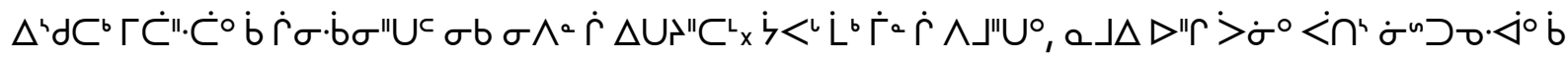
$\dot{\Gamma} \sigma \cdot \dot{b} \sigma^{\prime \prime} \cup c_{x}$

$\left.\dot{\Gamma} \circ \dot{b} \dot{r} s \dot{b} \dot{r}^{\iota} \nabla^{\circ} \dot{\Gamma} \circ \dot{b} \wedge\right\lrcorner " U^{\prime c} c_{x}$

"

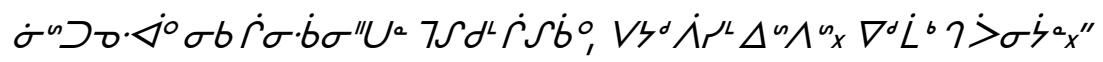

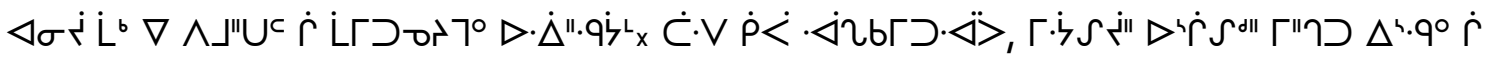

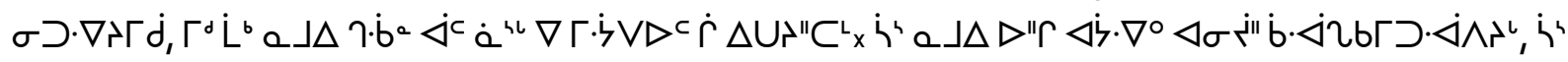




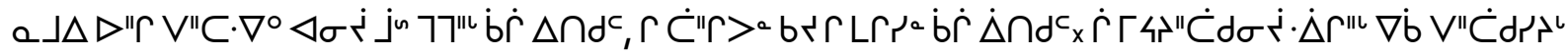

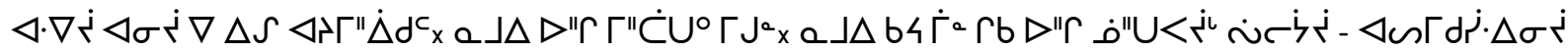

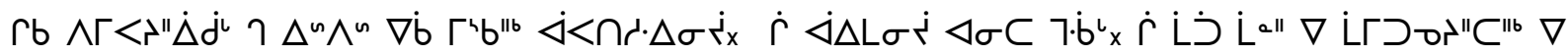

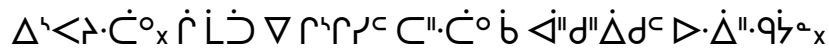

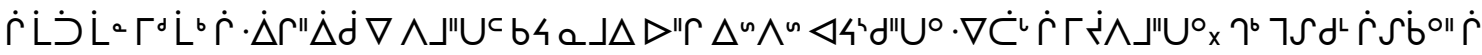

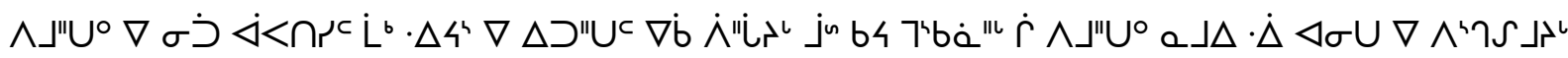

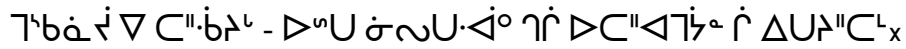

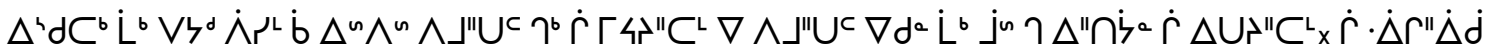
ר⿱亠凶禸

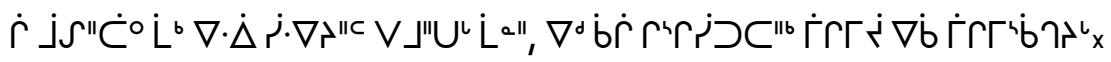

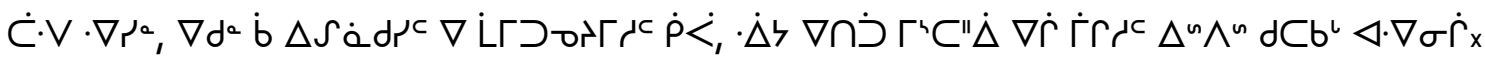

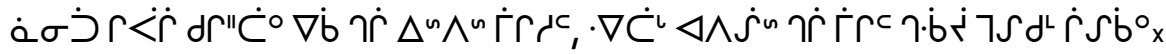

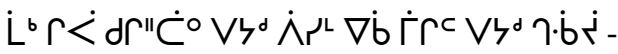

$. q \dot{\varphi} \dot{C} \cdot \vee r b \triangleleft \Delta L^{\circ}, \dot{\Gamma} \Delta U_{r}{ }^{\prime \prime} C^{L} \times$

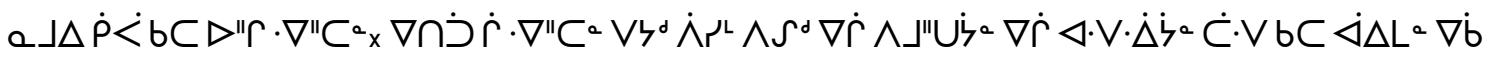

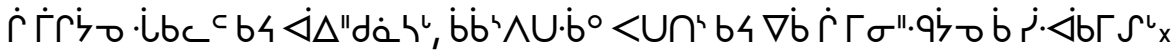

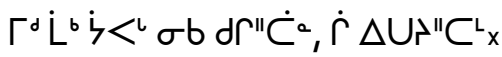

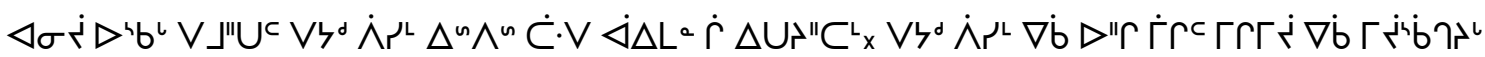

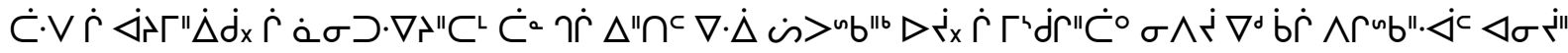

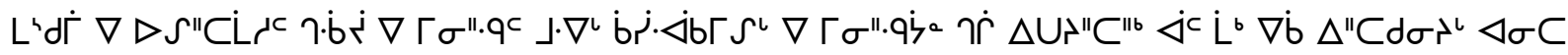

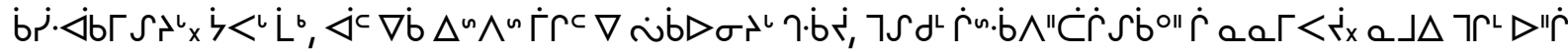

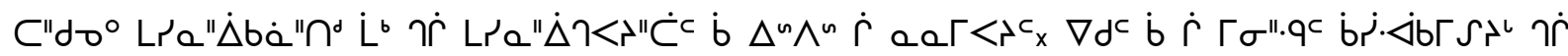

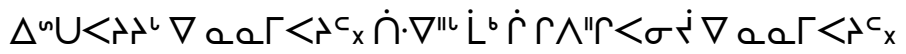

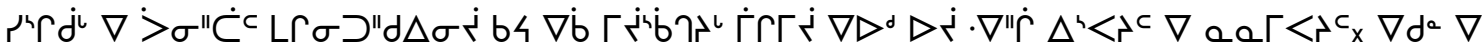

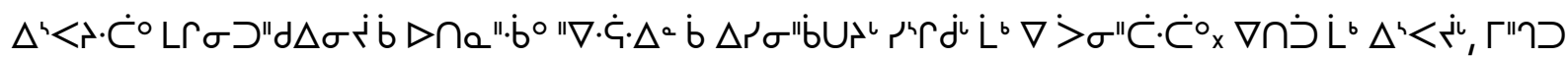

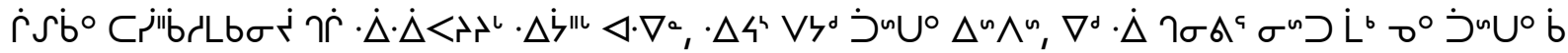

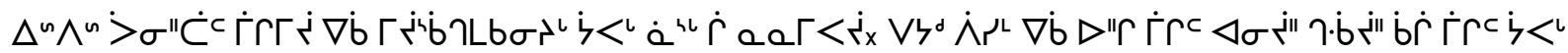

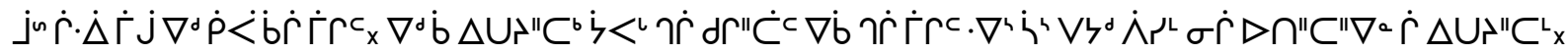

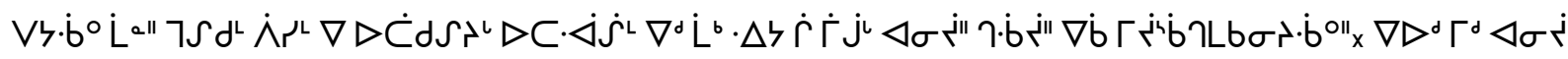




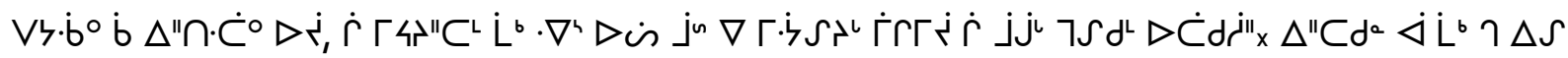

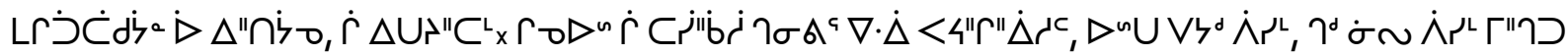

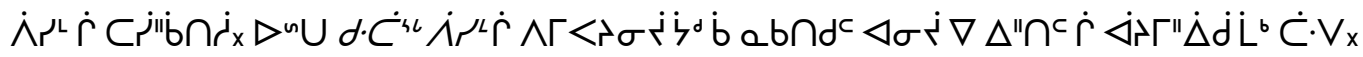

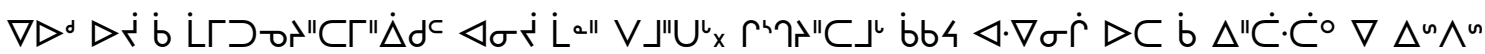

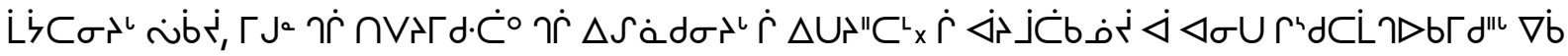

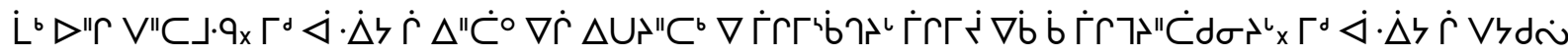

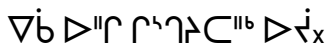

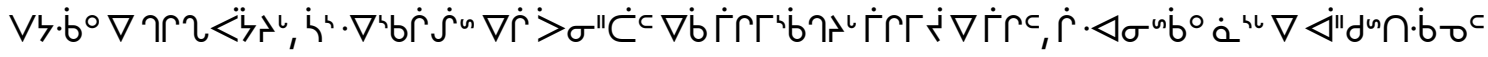

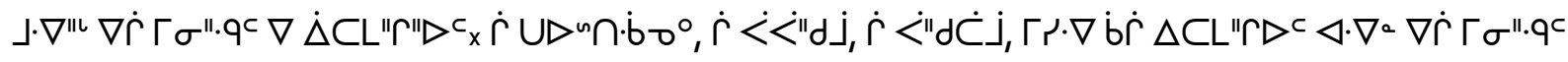
$\nabla d^{\circ} \dot{b} \Delta C L " r " \triangleright c^{c}$

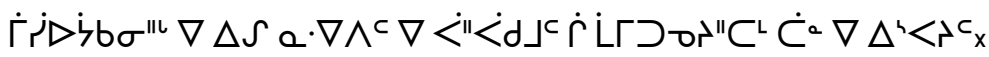

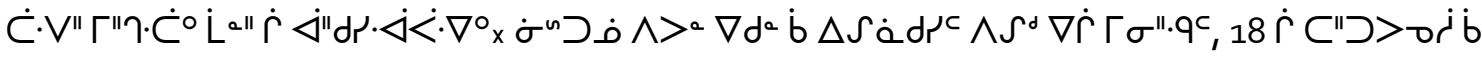

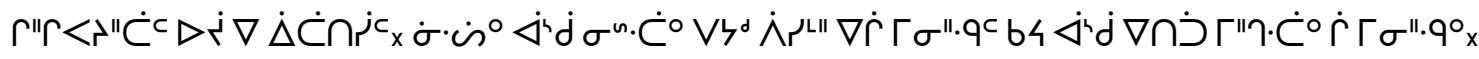

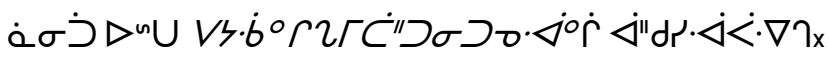

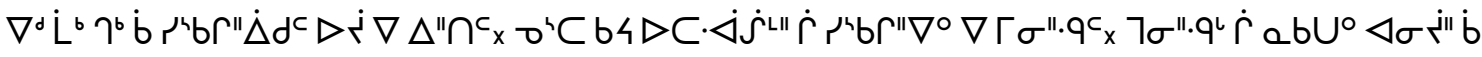

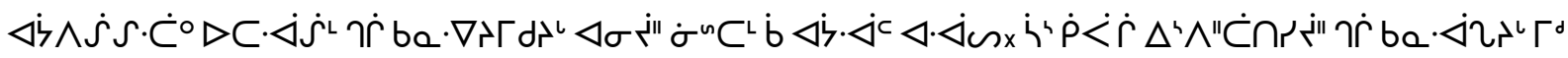

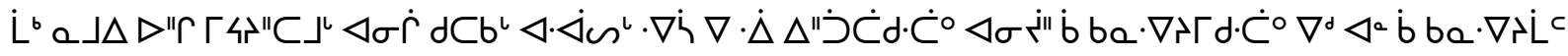

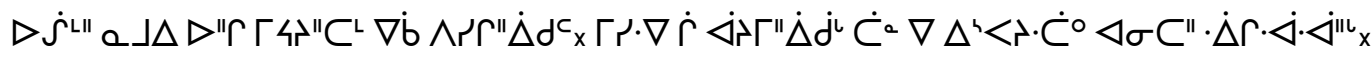

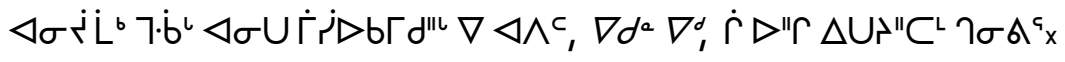

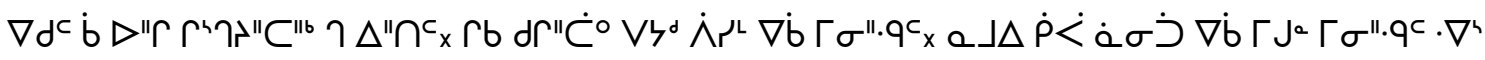

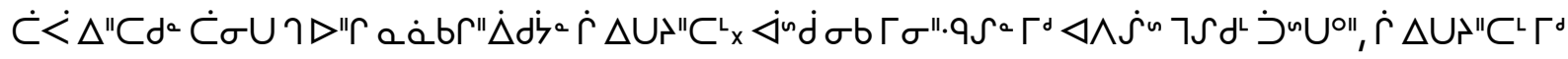

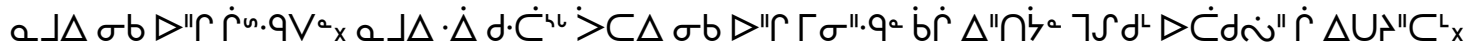

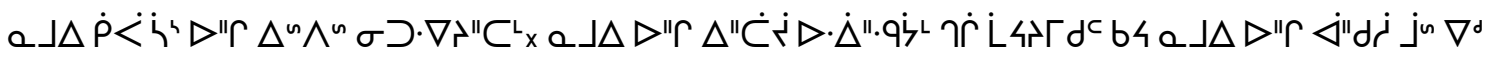

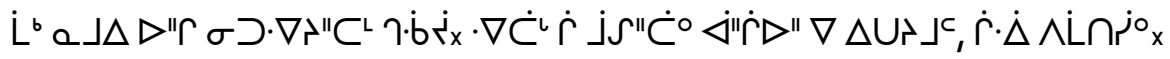

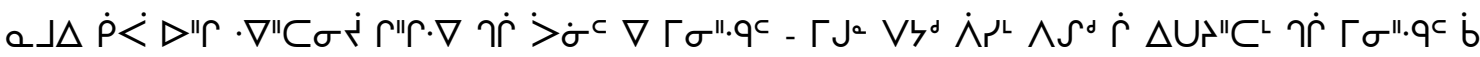

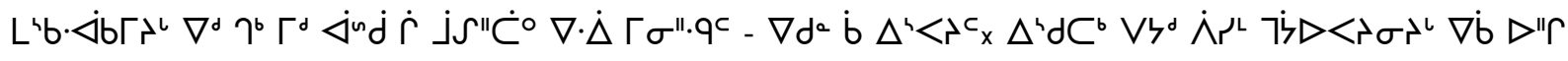

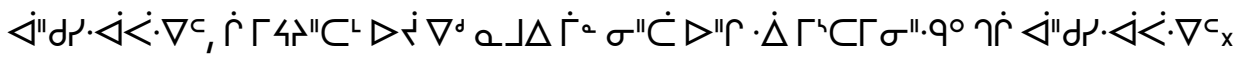




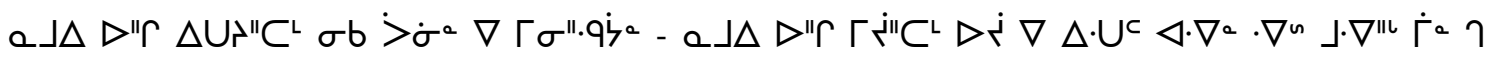

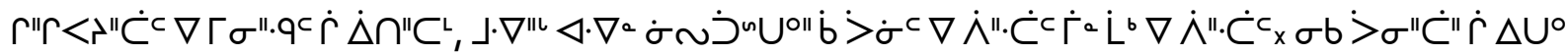
$\cdot \dot{\Delta} b \mathrm{x} U b^{n} \sigma \mathrm{b}>\sigma^{\prime \prime} \dot{C}^{a} \mathrm{x} \nabla d^{a} \nabla^{\mathrm{d} x}$ $\Delta U^{\prime \prime C} C^{L} x$

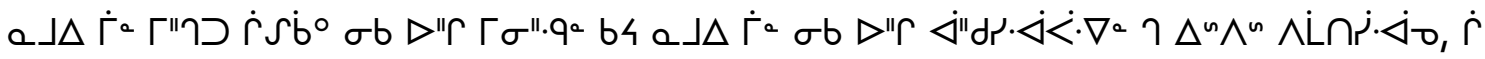

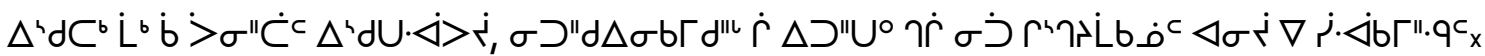

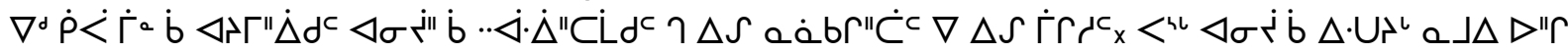

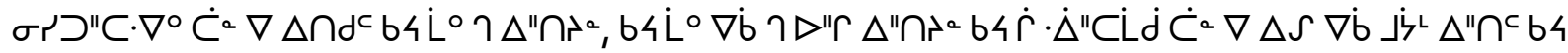

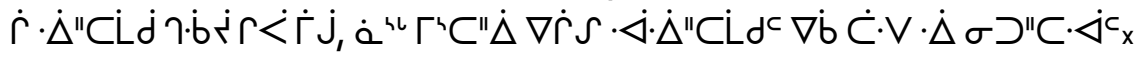

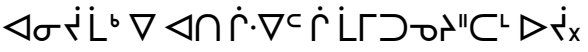

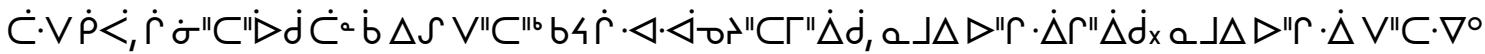

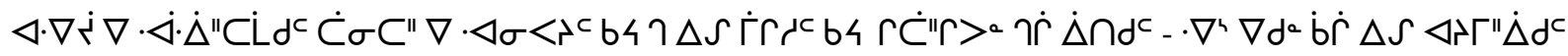

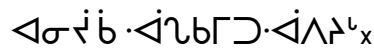

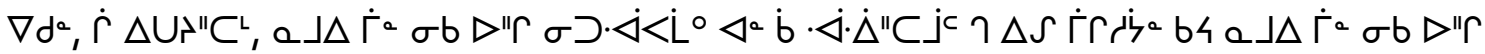

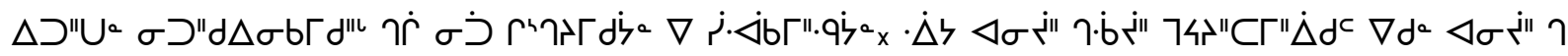

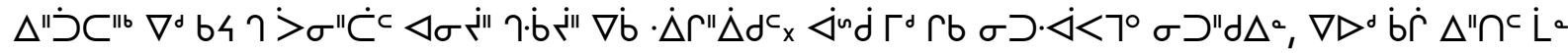

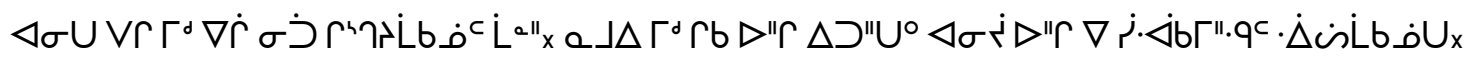

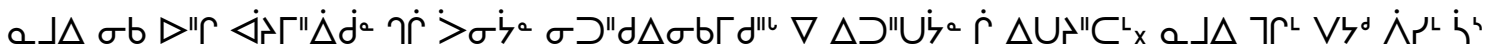

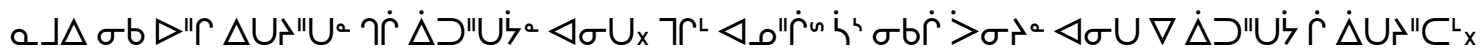

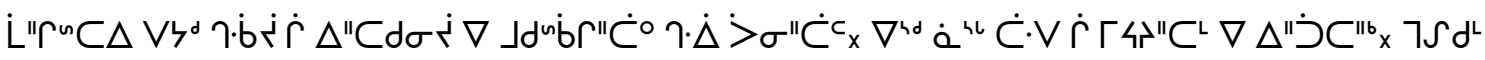

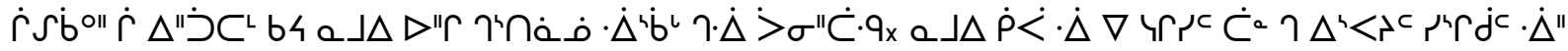

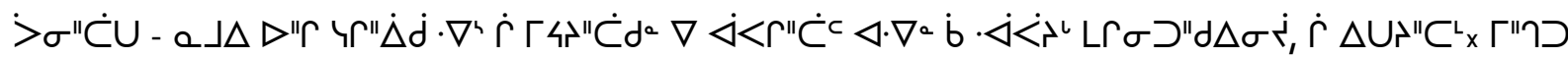

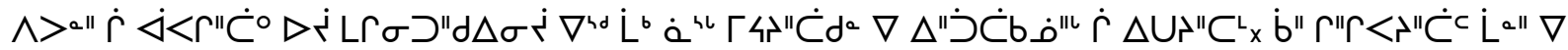

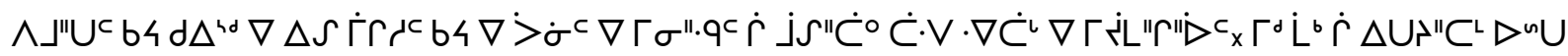

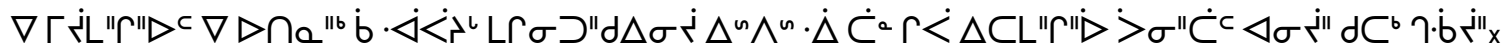

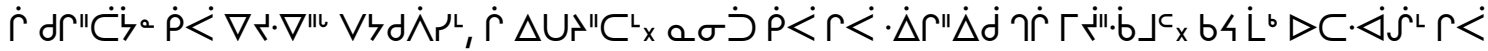

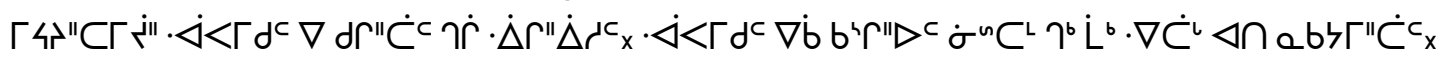

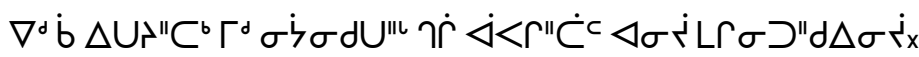




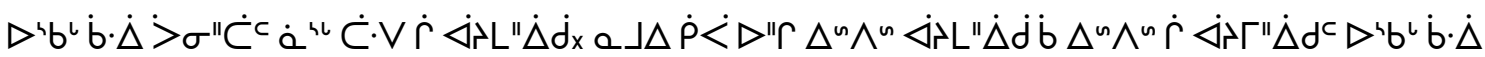

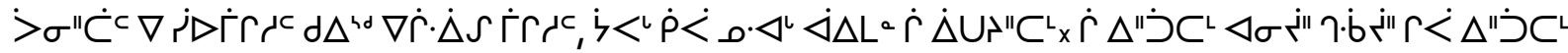

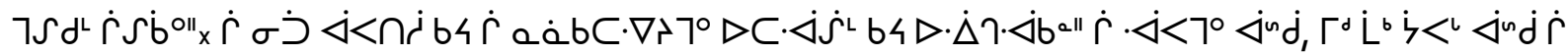

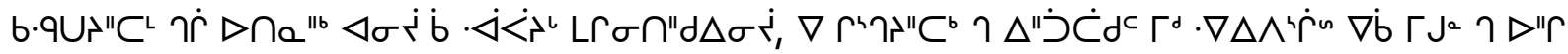

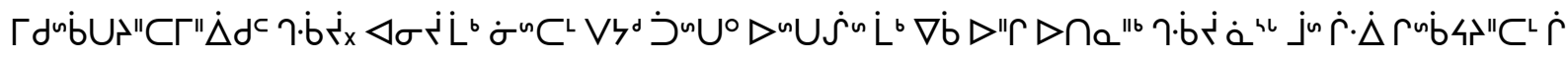

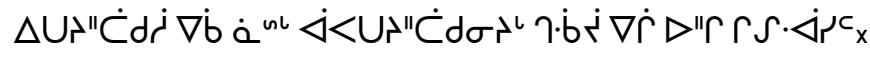

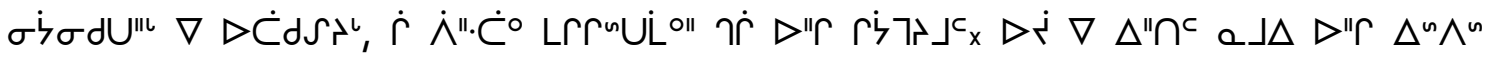

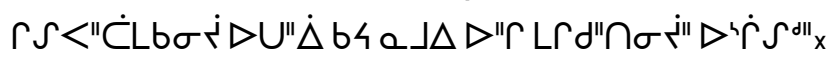

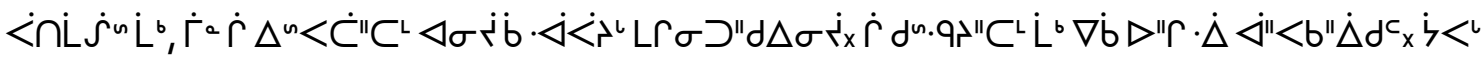

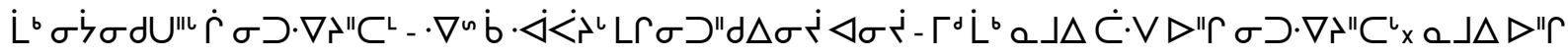

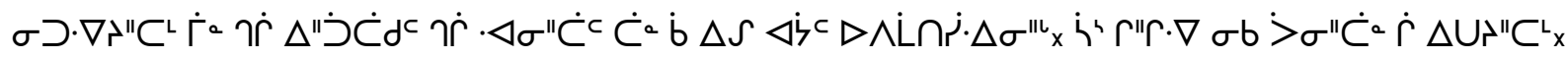

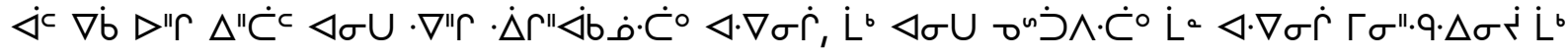

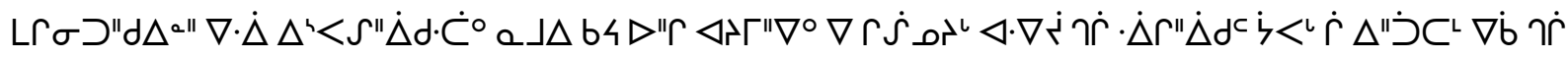

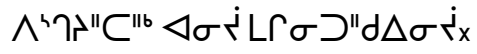

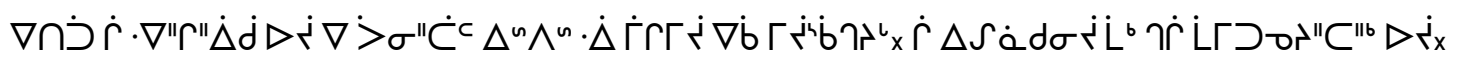

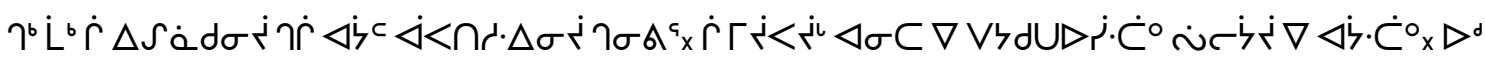

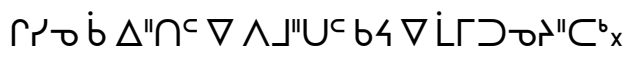

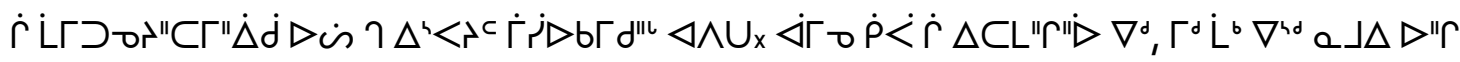

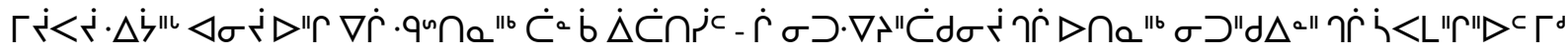

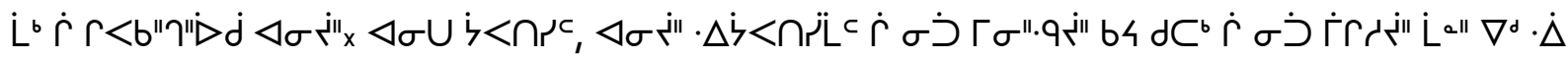

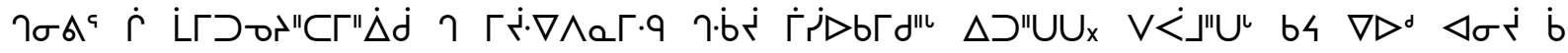

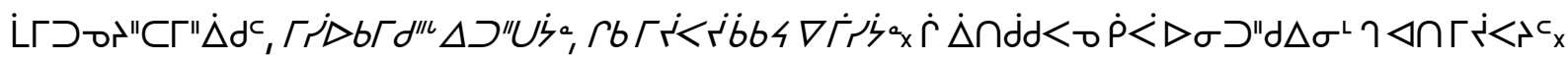
$\dot{C} \cdot \vee \dot{L}^{b} x$

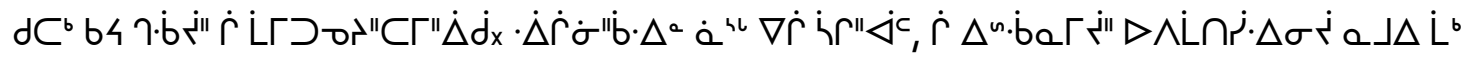

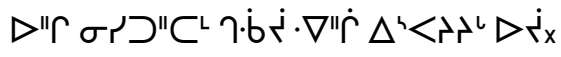

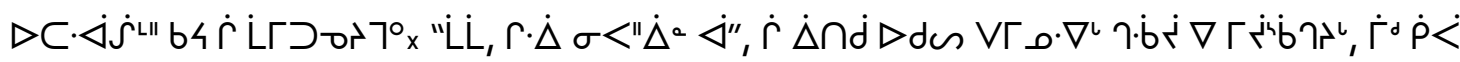

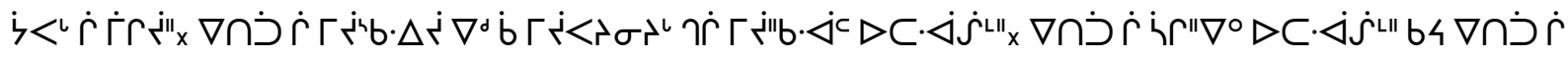
$\dot{s} \dot{\lambda} \cdot \nabla \dot{j}^{n} C \cdot \nabla^{\circ} x$

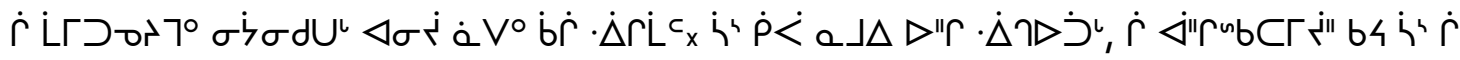

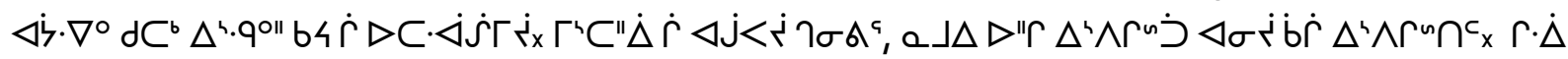

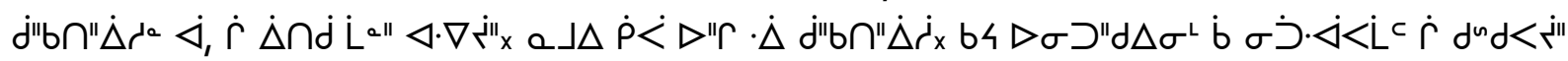




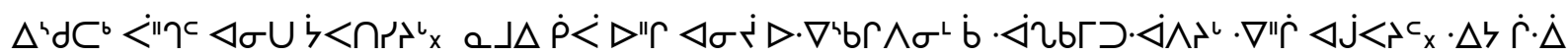

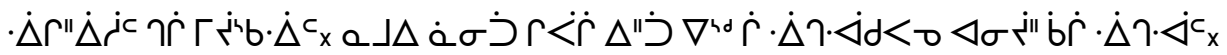

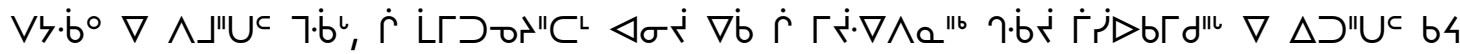

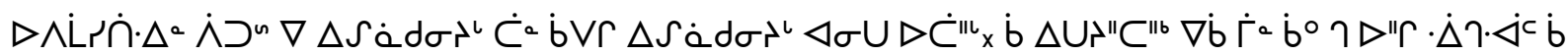

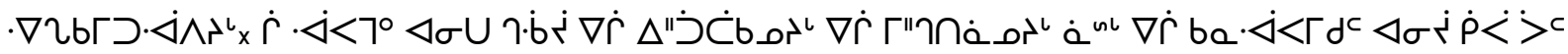

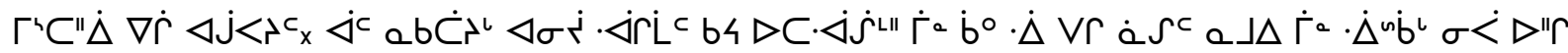
$\cdot \dot{\Delta} \cdot \dot{\triangleleft} \circ \dot{\Gamma} \Delta \mathrm{UP}^{\mathrm{N}} \mathrm{C}^{\mathrm{L} x}$

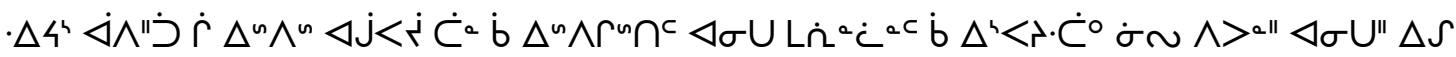

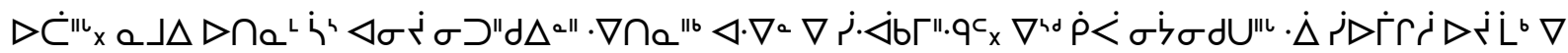

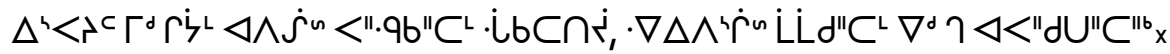

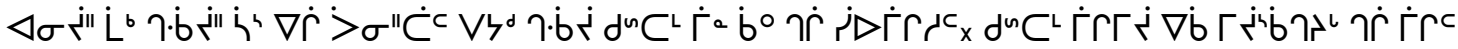

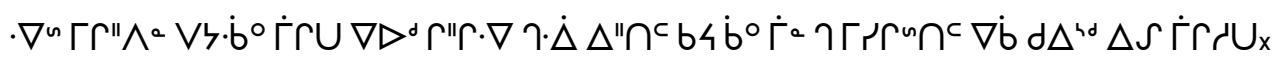

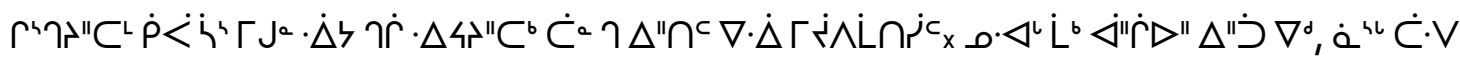

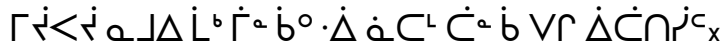

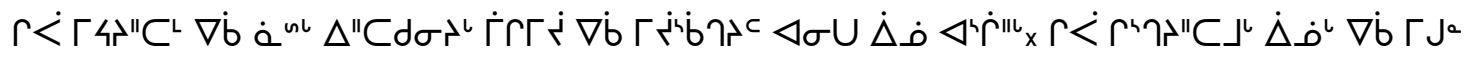

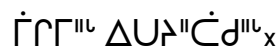

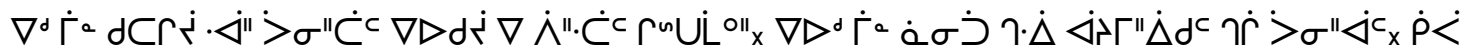

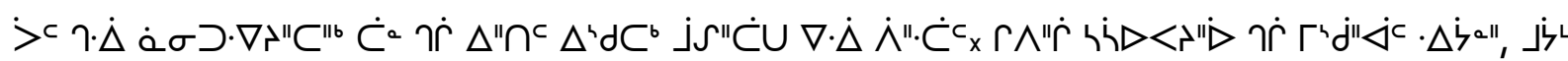

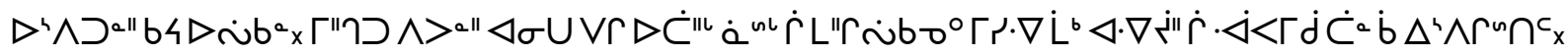

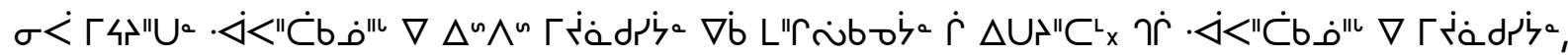

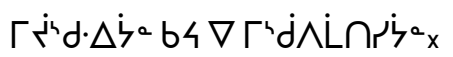

